\title{
A Pulsar Timescale Algorithm Using NANOGRAV Data
}

\author{
Dongshan YIN ${ }^{1,2,3, a}$, Shuhong ZHAO ${ }^{1,3, b}$,Yuping GAO ${ }^{1,3, c}$ \\ ,and Yuejuan JING ${ }^{1,2,3, d}$ \\ ${ }^{1}$ National Time Service Center, Chinese Academy of Sciences, Xi'an 710600, China \\ ${ }^{2}$ University of Chinese Academy of Sciences, Beijing 100049, China \\ ${ }^{3}$ Key Laboratory of time and frequency primary standard, National Time Service Center, Chinese \\ Academy of Sciences, Xi'an 710600, China

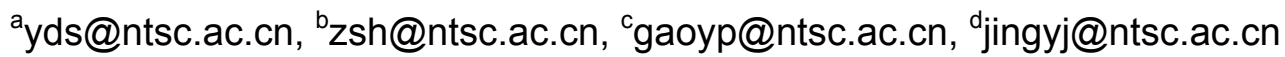

Keywords: pulsars timing residuals; ensemble pulsar time scale; frequency instability.

\begin{abstract}
Millisecond pulsar can generate another type of time scale that is totally independent of atomic time scale, because physical mechanisms of pulsar time scale and atomic time scale are quite different. Usually pulsar timing observation isn't even sampled, and their data set is sparse, So we use cubic spline interpolation to densify data file and make intervals between data points even, after that we employ vondrak filter to smooth data set and get rid of high frequency noise. We used the latest released NANOGRAV data to generate ensemble pulsar time. Clock difference between pulsar time(PT) and atomic time was got and instability of PT was analysed, RMS of clock difference is $0.056 \mu$ s, and long term frequency instability of $\mathrm{PT}(>1$ year $)$ is better than $4 \mathrm{E}-15$. Results show that instability of PT is comparable to that of atomic time scale.
\end{abstract}

\section{Introduction}

Nowadays high precision time measurement and service is mainly based on International Atomic Time(TAI) and Coordinated Universal Time(UTC), which are obtained from a combination of data from more than 400 atomic clocks kept by more than 70 timing labs, and calculated by BIPM with suitable algorithm. TAI is one version of terrestrial time(TT) generated by BIPM every month, TT(BIPM) is another realization of terrestrial time, which is generated by BIPM with one year long calibration data from primary frequency standards, that makes TT(BIPM) more accurate and stabler than TT(TAI). TT(BIPM) is generated once per year, which makes it inconvenient for real time service, but for pulsar timing observation TT(BIPM) is used as reference time.

Millisecond pulsars(MSP) exhibit excellent long-term rotation stability[1], which makes it suitable to generate another type of time scale that is totally independent of atomic time scale which is used as time standard worldwide. Physical mechanisms of pulsar time scale and atomic time scale are quite different. PT is also a realization of ideal time scale, which is accurate, stable, and measurable[1]. Accuracy of the best PT is comparable to terrestrial time(TT) in the world[2], so PT is suitable to be used to detect errors in international atomic time(TAI). The International Astronomical Union(IAU) time commission has started a working group on pulsar time scale, whose purpose is to carry out research on pulsar time scale and coordinate international cooperation in this field. Suitable algorithm for pulsar timescale should be designed especially because pulsar timing observation data is usually irregular and sampling rate is much lower than that of atomic clock comparison. In addition the model and timing noise for pulsar clock are different from atomic clock.

We used the latest released NANOGRAV data set to generate ensemble pulsar time scale, and analysed its instability, also compared its instability with atomic time scale's short-term and long-term instability.

\section{NANOGRAV Data Set}

The data set we used in this paper is NANOGRAV's 9 year timing data of 37 millisecond 
pulsars[3], which was observed with GBT and ARECIBO telescopes, detailed information of data set is listed in Figure. 1. PSR B1937+21 is excluded because it exhibits timing noise that is currently uncorrectable and is at a level significantly higher than white noise level. All residuals were generated with TEMPO2[4][5][6].

\begin{tabular}{|c|c|}
\hline 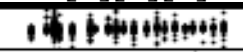 & 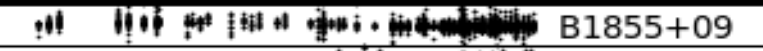 \\
\hline taliptit & 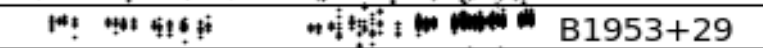 \\
\hline & J0023+0923 \\
\hline 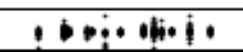 & J0030+0451 \\
\hline & J0340+4130 \\
\hline \multirow[t]{2}{*}{ 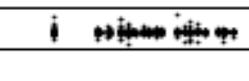 } & 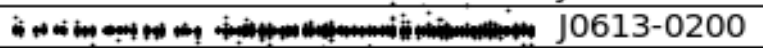 \\
\hline & J0645+5158 \\
\hline & J0931-1902 \\
\hline \multirow[t]{2}{*}{ 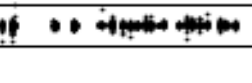 } & 1 $1012+5307$ \\
\hline & J1024-0719 \\
\hline \multirow[t]{3}{*}{$\cdot x+\infty$} & 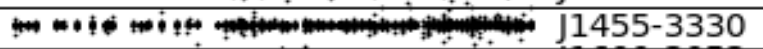 \\
\hline & 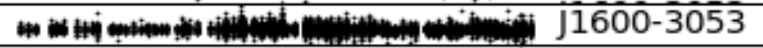 \\
\hline & 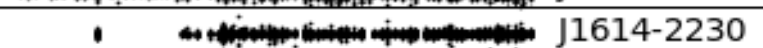 \\
\hline 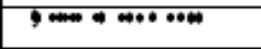 & 1 \\
\hline 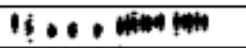 & 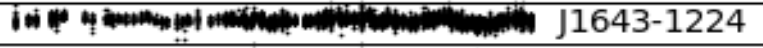 \\
\hline \multirow[t]{2}{*}{ 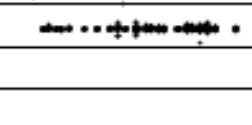 } & 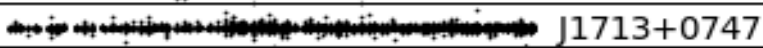 \\
\hline & 的 $1738+0333$ \\
\hline \multirow[t]{4}{*}{ - $\quad$ timatim } & 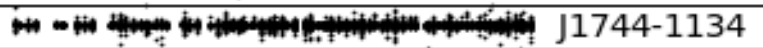 \\
\hline & J1747-4036 \\
\hline & 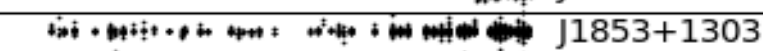 \\
\hline & J1903+0327 \\
\hline it $\quad$ ond in & 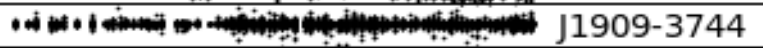 \\
\hline 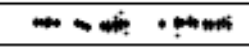 & 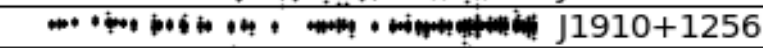 \\
\hline \multirow[t]{7}{*}{ 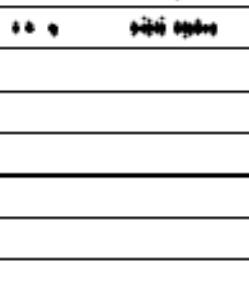 } & 19618-0642 \\
\hline & 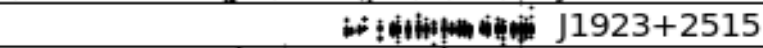 \\
\hline & 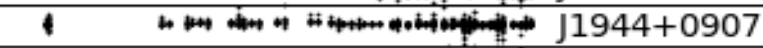 \\
\hline & J1949+3106 \\
\hline & J2010-1323 \\
\hline & J2017+0603 \\
\hline & 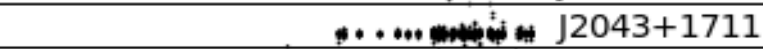 \\
\hline \multirow[t]{3}{*}{ 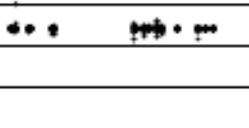 } & 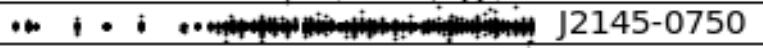 \\
\hline & 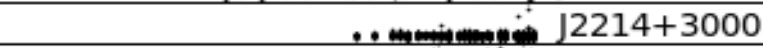 \\
\hline & 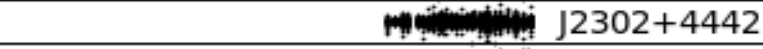 \\
\hline 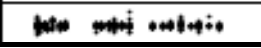 & 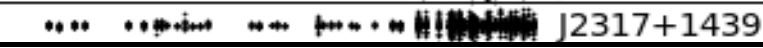 \\
\hline
\end{tabular}

Fig. 1 post-fit residuals of 36 MSPs from NANOGRAV data set, observation span from 53216(MJD) to 56599(MJD), all residuals was measured referred to TT(BIPM)

\section{Method}

Clock difference between pulsar time(PT) and atomic time(TT(BIPM)) is totally correlated between different pulsars[2], that means clock difference in different pulsars are exactly same, so we add post-fit residuals of $36 \mathrm{MSP}$ together to decrease the influence caused by other noise such as solar system ephemeris error, interstellar medium and timing noise. But it will increase signal to noise ratio (SNR) of measurement, weighted average of sum of 36 MSP's post-fit residuals is what we are interested in. As shown in Equation. 1,

$$
(A T-P T)=\sum_{i=1}^{36} \omega_{i}\left(A T-P T_{i}\right)
$$

where AT is reference atomic time, which is TT(BIPM) in pulsar timing observation, $\omega_{i}$ is weight of ith pulsar of all 36 pulsars, in order to decrease influence imposed by observation noise on PT, 
$\omega_{i}$ defined as below, where $R M S_{i}$ is ith pulsar's root of mean square of post-fit residuals.

$$
\omega_{i}=\frac{1}{R M S_{i}^{2}} \sum_{i=1}^{36} \frac{1}{R M S_{i}^{2}}
$$

In every pulsar's data file, there are bad data points. First we got rid of bad data points whose values are greater than 3 times of RMS( $3 \sigma$ principle). Usually clock difference is mainly composed of low frequency components, so we tried several filter methods(adaptive kalman, vondrak) to smooth observation datas first, results show that vondrak can remove high frequency noise and produce best clock difference data. As shown in Figure. 2 and Figure. 3,

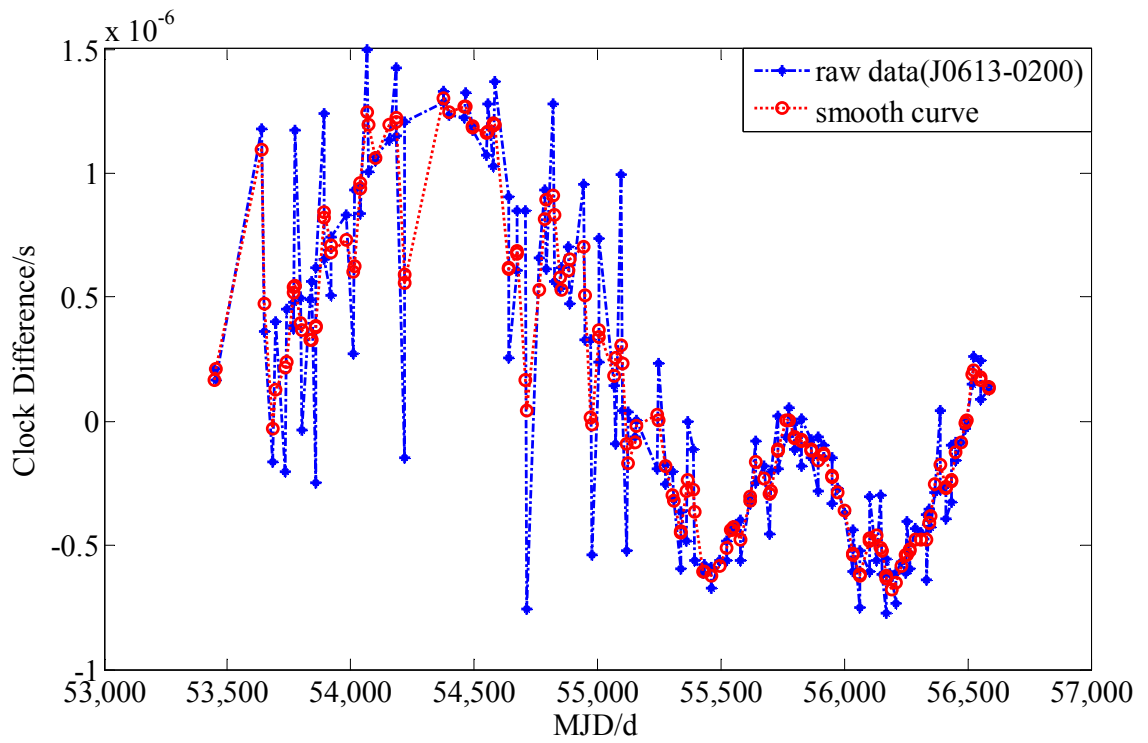

Fig. 2. before and after vondrak fit of pulsar J0613-0200

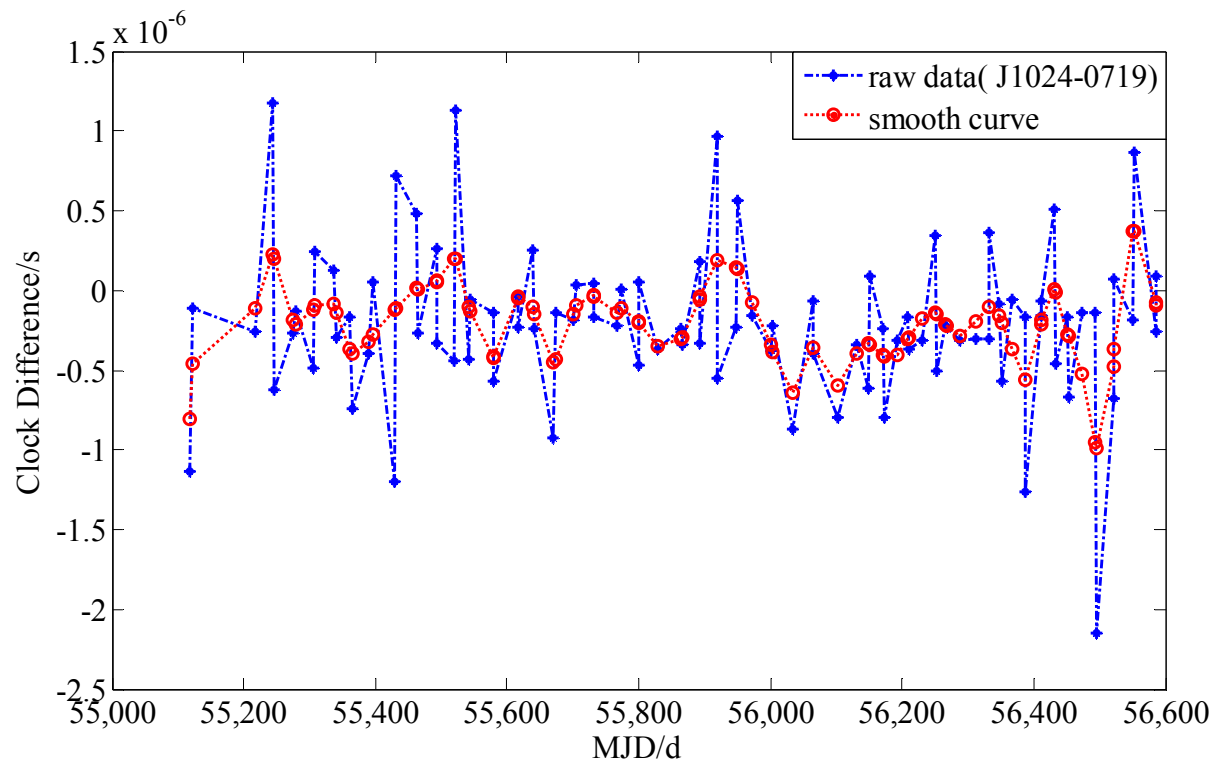

Fig. 3. before and after vondrak fit of pulsar J1024-0719

Almost all pulsars have sparse observation datas, and intervals between data points of each pulsar are not even. So we tried several interpolation methods to increase raw residuals data points, we tried polynomial interpolation(linear and quadratic polynomial interpolation) and cubic spline interpolation, results showed that cubic spline interpolation can provide best densified data set.

After all these steps, we got averaged post-fit residuals of 36 pulsars weighted by RMS of each pulsar's post-fit residuals, as shown in Figure. 2. 
Because of braking of pulsar rotation, pulsar time has apparent frequency drift rate, so we use overlapping variance to analyze its rotation instability, which uses all 3-sample combination of clock difference data.

$$
\sigma_{z}(\tau)=\frac{\tau^{2}}{2 \sqrt{5}}\left\langle c_{3}^{2}\right\rangle^{1 / 2}
$$

Equation.3 provides definition of $\sigma_{z}(\tau)$, usually it is used to evaluate frequency instability of pulsar time. In the equation $\tau$ is averaging time, \langle\rangle means weighted average over all subsets of the data, and $c_{3}$ is coefficient of cubic component of polynomial fit of each subset.

\section{Result And Disscussion}

Figure. 4 shows clock difference between pulsar time(PT) and atomic time(TT(BIPM)) with RMS $0.056 \mu \mathrm{s}$. At the very first beginning of the datas the fluctuation has greater amplitude, but after 2009 the amplitude of fluctuation is much smaller apparently. We think that is because the back-end instrument has been upgraded and more millisecond pulsars with smaller post-fit residuals were added(as shown in Figure. 1).

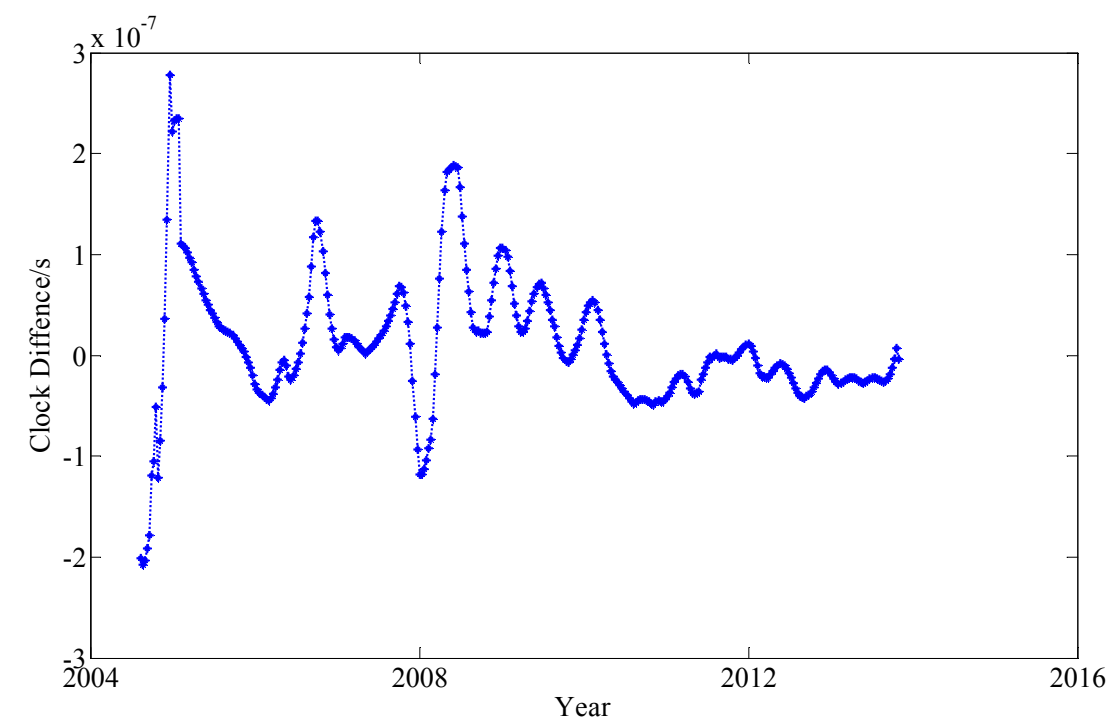

Fig. 4. clock difference between PT and atomic time referred to TT(BIPM)

The most common way to express the time domain instability of a time scale is by means of a sigma-tau plot which shows some measurements of frequency instability[7]. Figure. 5 shows frequency instability of PT, when sampling interval is smaller than 100 days the instability is greater than $1 \mathrm{E}-14$, but when sampling interval is greater than 100 days the instability is smaller than 1E-14[8]. Result shows that PT has good long term instability, but caused by high frequency observation noises and timing noise the short term instability of PT is not so good. 


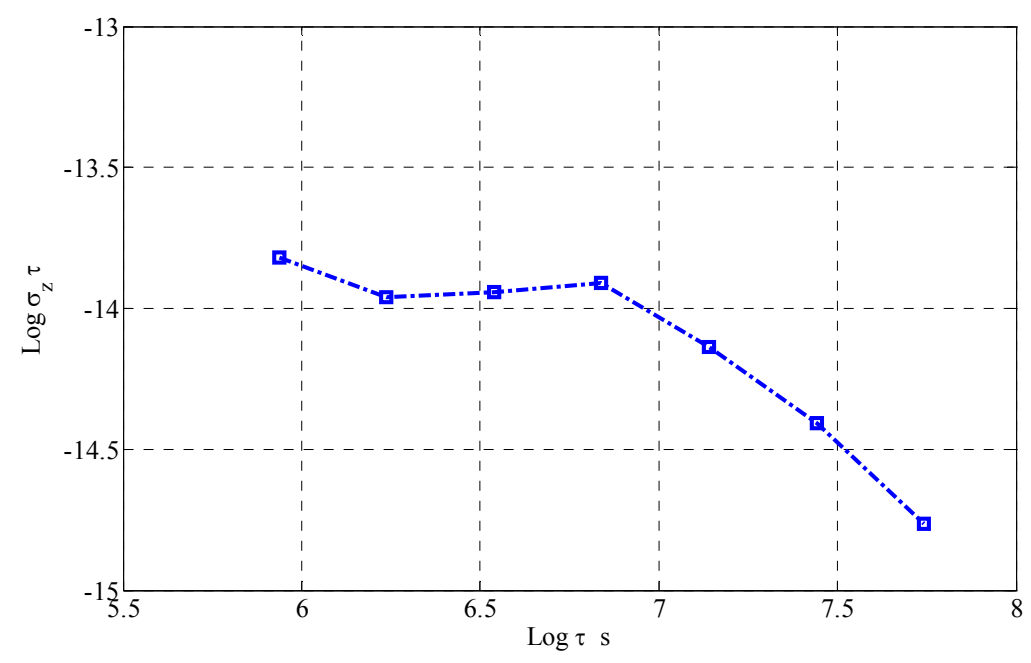

Fig. 5. Frequency instability of PT

\section{Summary}

From Figure. 5 we can tell when averaging time is shorter than 80 days, the main component of noise is frequency flicker noise and random walk frequency noise[7], we think this is caused by some system noise, such as pulsar rotation disturbance, observation system noise, or dispersion measurement fluctuate. But when averaging time is longer than 80 days, main component of noise is white frequency noise, which is typically observation noise. So in order to increase stability and accuracy of PT, accurater timing noise model, better observation instrument and longer data set are needed.

\section{Acknowledgement}

In this paper, the research was sponsored by the National Natural Science Foundation of China, (Project No. 11303032), the National Natural Science Foundation of China, (Project No. 11473029); and the Open Foundation for Key Laboratory of Geometric Information Engineering, (Project No. SKLGIE2013-M-1-1)

\section{References}

[1] Chongxia Zhong, Algorithm and Application of Ensemble Time Scale, Doctoral Dissertation,2007.

[2] G. Hobbs, W. Coles, R. N. Machester, arXiv: 1208.3560v1 [aetro-ph.IM] [J] ,2012.1-7.

[3] Arzoumanian et al. The NANOGrav Nine-year Data Set: Observations, Arrival Time

Measurements, and Analysis of 37 Millisecond Pulsars[J], arXiv:1505.NNNN,2015.

[4] G. Hobbs, R. T. Edwards, R. N. Manchester, MNRAS, 2006, 369, 655.

[5] G. Hobbs et al., MNRAS, 2009, 394, 1945.

[6] P.G. Clem, M. Rodriguez, J.A. Voigt and C.S. Ashley, U.S. Patent 6, 2001,231, 666.

[7] G. Hobbs, A. Archibald, Z. Arzoumanian, D. Backer, M. Bailes, N. D. R. Bhat, M. Burgay, et al., Classical and Quantum Gravity, 27(8), 2006.

[8] Riley W J. Handbook of frequency stability analysis.National Institute of Standards and Technology Special Publication 1065. Washington: U. S. Government printing office,2008. 